\title{
Guides de mémoire et stratégies discursives
}

\author{
Anna Giaufret ${ }^{1, *}$, Stefano Vicari ${ }^{2}$ \\ ${ }^{1}$ Dipartimento di Lingue e culture moderne, Università di Genova \\ ${ }^{2}$ Dipartimento di Lingue e culture moderne, Università di Genova
}

\begin{abstract}
Résumé. Cette étude souhaite contribuer à la compréhension des mécanismes discursifs à travers lesquels se construit et se diffuse dans l'espace discursif public le discours de la mémoire des deux conflits mondiaux du $\mathrm{XX}^{\mathrm{e}}$ siècle. Notre corpus est constitué de 10 guides touristiques de mémoire publiés en France à l'occasion du Centenaire de la Première Guerre Mondiale (Michelin, Routard, Petit Futé).
\end{abstract}

\begin{abstract}
Memory guides and and discursive strategies. This study aims at contributing to the understanding of discursive strategies through which memory discourse on the two XX century World Wars is built. Our corpus is made of ten memory tourist guides published in France on the occasion of the Centenary of the World War I (Michelin, Routard and Petit Futé).
\end{abstract}

\section{Introduction}

Cette étude a l'objectif de contribuer à la compréhension des mécanismes discursifs à travers lesquels se construit et se diffuse dans l'espace public le discours de la mémoire des deux conflits mondiaux ayant ravagé le territoire européen au cours du $\mathrm{XX}^{\mathrm{e}}$ siècle. Afin d'y parvenir, nous allons analyser un corpus de dix guides touristiques de mémoire publiés en France à l'occasion du Centenaire de la Première Guerre Mondiale. Nos analyses se situent dans le cadre d'un projet de recherche plus vaste, MemWar, où littéraires et linguistes de différentes langues-cultures de l'espace européen s'attachent à analyser, d'un côté, les représentations artistico-littéraires des deux guerres mondiales et de la guerre d'Espagne (BD, romans, etc.) au $\mathrm{XXI}^{\mathrm{e}}$ siècle et, de l'autre, les différents types de discours circulant dans la presse et dans le discours touristique ${ }^{\mathrm{i}}$.

Le choix du discours touristique se justifie en effet par l'ampleur et la diffusion dont ce qu'on appelle désormais «tourisme de mémoire » (Hertzog, 2012) témoigne ces dernières années, en Europe et surtout en France, le pays le plus riche en termes de patrimoine mémoriel lié aux conflits mondiaux du $\mathrm{XX}^{\mathrm{e}}$ siècle. Promue et développée, du moins à partir de 2012, sous l'égide conjointe du Ministère de la Défense, de celle du Tourisme français et des collectivités territoriales, la visite des sites qui ont vu le déploiement des deux guerres

\footnotetext{
* Corresponding author : anna.giaufret.unige@gmail.com. Anna Giaufret a rédigé les paragraphes 1, 2, 4.1 et 4.3. Steafno Vicari a rédigé les paragraphes 3, 4.2. et la conclusion.
} 
mondiales connait en effet un succès croissant auprès d'une clientèle internationale, d'origine principalement européenne (ministères de la Défense et du Tourisme, 2012). L'Agence de développement touristique de la France (Atout France) considère désormais ce type de tourisme comme « un enjeu stratégique majeur pour le tourisme français : faire émerger une offre touristique d'excellence transformant des territoires de mémoire en destinations touristiques de mémoire et d'histoire » (Atout France, en ligne).

Dans ce contexte, les principales maisons d'édition de tourisme et voyage françaises n'ont pas tardé à publier des guides permettant aux touristes de découvrir les lieux dits « de mémoire » : c'est ainsi que Michelin réédite 6 guides sur les champs de bataille en France (la première édition a été publiée entre 1917 et 1922), et que Routard et Petit Futé se lancent pour la première fois dans cette entreprise, comblant ainsi un vide dans le panorama des publications touristiques destinées au grand public en France.

A la suite de la présentation de ces guides et des références théoriques et méthodologiques, qui se situent principalement dans le cadre des travaux sur l'hétérogénéité énonciative et le discours rapporté (Authier-Revuz, 1984, 1995, Rosier, 2008, Barthelmebs-Raguin, et al., 2018), nous montrerons l'intérêt d'analyser les formes de discours rapporté et les fonctions qu'elles remplissent dans ces guides et, notamment, leur rôle dans la construction d'un discours de la mémoire partagée des conflits mondiaux.

\section{Objectifs et problématique}

Le discours de mémoire des guides touristiques se fonde sur un oxymore (voire une aporie) constitutif : il s'agit de traiter des évènements traumatiques du passé, d'en décrire les ruines, les restes, tout en mettant entre parenthèses leur dimension éminemment dysphorique, dimension qui ne peut être déployée dans toute son intensité que dans le but de susciter le pathos chez le lecteur, et donc une identification prothétique avec les victimes. C'est pour cette raison que les guides doivent faire un travail de reconfiguration des lieux des guerres, qui sont aujourd'hui devenus des lieux radicalement différents de leur état au moment des événements qu'ils relatent. Il s'agit d'un travail de transformation radicale du dysphorique en euphorique, d'une mémoire qui évoque le traumatisme, la souffrance, la mort en un désir de visiter les traces de ce passé funeste afin de revivre ces émotions pour traverser un véritable processus de catharsis afin de se purger du mal et faire en sorte que tout cela ne se répète plus jamais.

Par ailleurs, les lieux de mémoire sont aussi liés à des sentiments euphoriques qui ne sont plus vraiment de mise aujourd'hui, comme le patriotisme, l'héroïsme, et tout ce qui a contribué à envoyer au massacre des millions de combattants. Sans parler de leurs avatars encore moins nobles lorsqu'ils sont liés au régime de Vichy ou à la France collaborationniste.

Comment les guides arrivent-ils à maintenir cet équilibre précaire entre dysphorie et euphorie, entre adhésion et prise de distance, afin de satisfaire un public aussi large que possible? C'est ce que nous allons tenter d'expliquer dans cette contribution.

Les guides rentrent en effet de plein droit dans le discours de mémoire tel qu'il se diffuse à notre époque, puisque :

1- Ce sont des textes créés pour le Centenaire de la Première Guerre Mondiale visant à diffuser et promouvoir un discours mémoriel des Guerres Mondiales à travers la valorisation des lieux de la guerre, qui revêtent aujourd'hui une importance stratégique dans le panorama touristique français - ainsi que nous l'avons expliqué dans l'introduction ;

2- Ce sont des textes emblématiques de la façon dont le discours de mémoire des Guerres Mondiales se construit et se diffuse dans le tissu social d'aujourd'hui, ainsi que des textes à grande diffusion qui touchent toutes les couches de la population. 
Alors que les guides Michelin sont d'habitude classés dans la catégorie des guides culturels et le Routard et le Petit Futé dans celles des guides pratiques, la nature particulière des guides de mémoire estompe en partie ces différences (pour une catégorisation des guides, voir Lugrin 2004 : 237, Rebeyrolles 2004 : 178);

3- Ce sont des textes fondés essentiellement sur les témoignages, les traces, les documents (photos, discours, textes, etc.) assurant l'authenticité du récit historique, leur relation étroite avec l'histoire, l'historiographie et la conservation du patrimoine, tout en s'appuyant sur le pathos.

Cela justifie donc notre hypothèse de départ, à savoir que l'inscription en discours de différentes formes d'hétérogénéité énonciative constitue l'un des moyens discursifs privilégiés par lesquels se construit et sur lequel repose le discours de mémoire des guerres, cette hétérogénéité remplissant plusieurs fonctions que nous nous proposons de relever.

\section{Corpus et remarques méthodologiques}

\subsection{Guides touristiques de mémoire et hétérogénéité énonciative}

Les guides de mémoire contemporains en France sont au nombre de dix, dont six ont été publiés par Michelin: Les Champs de bataille - La Marne et la Champagne (2014, abrégé en MMC) ; Verdun, Argonne, Saint-Mihiel (2016, abrégé en MVAS) ; Alsace Moselle : Les combats des Vosges (2014, abrégé en MAM) ; Flandres Artois : Ypres, Nord, Pas-deCalais (2013, abrégé en MFA) ; Le Chemin des Dames : Aine, Oise (2014, abrégé en MCD) ; Somme : Amiens, Péronne, Albert (2016, abrégé en MS). Le Routard en a publié trois, dont un sur les lieux de la Seconde Guerre Mondiale : Picardie 14-18. Centenaire d'un conflit mondial (2013, RP) ; Le Débarquement \& la bataille de Normandie (2014, RD) ; Grande Guerre 14-18. Les chemins de mémoire. Des Flandres aux Vosges (2015, RCM). Enfin, Petit Futé a publié en 2016 un Guide des lieux de mémoire (PF), qui présente les lieux symboles de l'histoire française, à partir de l'époque napoléonienne jusqu'à la Seconde Guerre Mondiale.

Dans le but de vérifier notre hypothèse concernant la centralité du rôle joué par l'hétérogénéité énonciative dans la construction du discours de mémoire dans les guides touristiques, nous nous sommes appuyés principalement sur les études linguistiques et énonciatives d'Authier-Revuz (1984, 1995) et, plus récemment, de Rosier (2008). En nous inscrivant dans le sillage de ces travaux, nous avons effectué une recherche automatique sur l'ensemble du corpus des indices de ce qu'on appelle à la suite d'Authier-Revuz (1984) « hétérogénéité énonciative montrée », à savoir des "formes linguistiques représentant des modes divers de négociation du sujet parlant avec l'hétérogénéité constitutive de son discours » (Authier-Revuz, 1984 : 99). Nous nous sommes donc attachés à repérer, classer et analyser les formes d'hétérogénéité tant au niveau de la macrostructure des guides qu'au niveau de la microstructure.

Nous nous sommes aussi inspirés des travaux sur la communication touristique (notamment Baider, Burger, Goutsos 2004) ainsi que sur les guides de mémoire (notamment Kottelat, 2015).

\subsubsection{La macrostructure}

Au niveau macrostructurel, en effet, les guides Michelin et Routard comprennent des parties entières signées par des auteurs autres que les éditeurs. Et notamment, les guides Michelin présentent une stratification discursive particulière: la deuxième section 
" Comprendre la Grande Guerre » compte une trentaine de pages et est identique dans tous les guides. Signée par François Crochet, historien et spécialiste de la Première Guerre Mondiale, elle constitue une longue introduction historique présentant les évènements principaux de la guerre, les causes, les effets, les champs de bataille et, enfin, le tourisme de mémoire aujourd'hui. L'auteur n'oublie pas de spécifier dans la conclusion de son texte non seulement le premier projet éditorial de la maison Michelin, mais aussi les sources d'où elle avait tiré les renseignements contenus dans ses guides, à savoir les Journaux des marches et des opérations (JMO) :

C'est en ce sens que cette nouvelle collection de guides Michelin qui s'appuie sur celle des années d'après-guerre prend toute sa valeur. À l'époque, des équipes complètes avaient fait un vrai travail d'historien, consultant les documents militaires et notamment les Journaux des marches et d'opérations (JMO) des unités, afin de les suivre au jour le jour. Ces renseignements nous sont encore précieux aujourd'hui. Mais les guides offrent aussi une présentation mémorielle qu'il convient aujourd'hui d'actualiser et de contextualiser (MCD 2014 : 52).

Cautionner les contenus des guides d'autrefois mais surtout en justifier la réédition contemporaine : voici les deux objectifs principaux de cette section qui, par la mise en avant de l'autorité de l'historien et des sources historiques dûment relues et contextualisées, garantit la fiabilité du discours véhiculé dans les parties descriptives qui suivent. L'entrelacement des voix se complique ultérieurement dans ces guides dans la mesure où les sections de présentation des lieux sont ponctuées par l'insertion d'images des vieux guides Michelin toujours suivies d'un encadré les recontextualisant ou prolongeant les renseignements qui y sont contenus, comme par ailleurs cela est signalé par l'équipe éditoriale dans la préface de tous les guides :

Cette nouvelle collection a pour ambition de présenter ces initiatives qui se multiplient. Les 6 titres qui couvrent l'ensemble du front permettent de mesurer l'évolution des sites et des paysages dans les régions marquées par la guerre. Des extraits des guides d'époque et des illustrations contemporaines témoignent de ces changements (MMC $2014: 3$ ).

En ce qui concerne les autres guides, si le Petit Futé ne présente pas de sections signées par d'autres auteurs, les guides Routard sont préfacés eux-aussi par des historiens et/ou des personnalités impliquées dans la gestion et la promotion du tourisme de mémoire. Cela est vrai pour les deux guides de la Première Guerre Mondiale, alors que pour le guide de la Seconde, la préface est signée par le directeur de la maison d'édition, Philippe Gloaguen. C'est ainsi que l'on peut lire dans le RP et dans le RCM, respectivement :

À l'aube du XXIe siècle, l'actualité quotidienne ne nous invite-t-elle pas à réfléchir à la nécessité de privilégier l'échange pour une meilleure compréhension des individus entre eux ? Puisse donc le tourisme de mémoire contribuer à la rencontre entre générations du monde entier sur les terres de l'Aisne, de l'Oise et de la Somme. En Picardie.

Carl Ooghe, Guide international des champs de bataille 1914-1918

Matthias Prohl, Directeur du Collège de Metzingen - Ville jumelée avec Noyon

Arlène King, Directrice du Mémorial Terre-Neuvien de Beaumont-Hamel (RP $2013: 5$ )

Grace au parcours très complet proposé dans ce guide, le visiteur trouvera tout ce qu'il faut pour découvrir la Grande guerre in situ, mais également s'interroger sur les souffrances endurées, et sur bien d'autres questions. 
Qu'il n'hésite pas aussi, à quitter les sentiers battus, pour se promener dans ces immenses zones de mémoire, où le souvenir affleure de manière inattendue et souvent émouvante.

Nicolas OFFENSTADT Université Paris-1 Panthéon-Sorbonne 1

Membre du Conseil scientifique de la Mission du centenaire de la Première Guerre mondiale (RCM $2015: 7$ )

Ces quelques lignes de conclusion des préfaces des deux guides montrent bien qu'elles remplissent à peu près la même fonction que chez Michelin : légitimer le discours tenu par le guide du point de vue historique tout en promouvant la nécessité du tourisme de mémoire. Cela est montré également par le fait que les signatures sont accompagnées, là où c'est le cas, des prestigieuses appartenances institutionnelles des auteurs. Bien que déclinée de manière différente dans les guides Michelin et Routard, la stratification discursive caractérisant la macrostructure de presque tous les guides analysés semble un trait caractérisant ce type de textes touristiques sui generis, où au plaisir de la visite et à la fonction d' « invitation au voyage », promotionnelle, propre de tous les guides touristiques (Kerbrat-Orecchioni, 2004) se superpose la dimension historico-mémorielle, reposant sur un souci de précision et de fiabilité des sources relatées.

\subsubsection{La microstructure}

$\mathrm{Au}$ niveau de la microstructure, nous avons recherché les formes de l'hétérogénéité énonciative dans les parties descriptives des lieux, les encadrés ainsi que dans les pages consacrées à l'approfondissement de certains sujets ou évènements. Ces formes sont représentées par des marques typographiques (guillemets et tirets) et par des verbes introducteurs des formes canoniques de discours rapporté (Rosier, 2008 : 56), comme les verba dicendi les plus communs (dire, annoncer, rappeler), exprimant parfois une modalisation (estimer), les verba scribendi (écrire, lire), les verbes d'attitude propositionnelle (croire, penser, trouver) et d'autres verbes métalinguistiques comme désigner, nommer et appeler. Si l'éventail n'est pas exhaustif, il est suffisamment large pour dresser un panorama des formes les plus utilisées dans ce corpus pour marquer la voix de l'autre en discours. La recherche a permis d'identifier au total 848 formes ainsi réparties selon les guides :

Tableau 1. Nombre de formes d'hétérogénéité énonciative dans chaque guide

\begin{tabular}{|l|c|c|c|c|}
\hline Guides & Totaux & Dénominations & Discours direct & $\begin{array}{c}\text { Autres formes } \\
\text { Discours indirect, discours narrativisé }\end{array}$ \\
\hline MAM & 105 & $51(49 \%)$ & $43(45 \%)$ & $11(6 \%)$ \\
\hline MVAS & 85 & $45(53 \%)$ & $37(44 \%)$ & $3(<3 \%)$ \\
\hline MFA & 83 & $46(55 \%)$ & $23(28 \%)$ & $14(17 \%)$ \\
\hline MMC & 79 & $31(39 \%)$ & $44(60 \%)$ & $4(1 \%)$ \\
\hline MS & 79 & $39(49 \%)$ & $38(48 \%)$ & $2(3 \%)$ \\
\hline MCD & 74 & $39(53 \%)$ & $24(32 \%)$ & $11(15 \%)$ \\
\hline RGG & 142 & $55(39 \%)$ & $70(49 \%)$ & $8(12 \%)$ \\
\hline RD & 68 & $28(41 \%)$ & $32(47 \%)$ & $10(16 \%)$ \\
\hline RP & 64 & $24(38 \%)$ & $30(47 \%)$ & $10(14 \%)$ \\
\hline PF & 69 & $36(52 \%)$ & $23(33 \%)$ & $17 \%)$ \\
\hline
\end{tabular}

Comme on peut le constater, les marques d'hétérogénéité énonciative concernent surtout deux phénomènes distincts. D’abord, des dénominations dont les auteurs montrent, voire 
exhibent, une origine énonciative autre, préexistante à la mise en discours et/ou le processus même de dénomination. Comme on le verra dans les analyses, ces dénominations, loin de représenter des étiquettes stabilisées en langue, montrent bien « une certaine dynamique discursive, qui engage tout à la fois la perception, la mise en discours, et l'appréhension énonciative de la mise en mots » (Longhi 2014 : en ligne) et, par-là, permettent de dégager toute une série de stratégies discursives adoptées dans ces guides afin de construire un discours de la mémoire à la fois fiable et partagé par la communauté des locuteurs. Ensuite, les citations directes de différentes personnalités, suivant des tendances variées selon les typologies de guides et les enjeux des discours. Voici deux exemples concernant ces deux phénomènes :

\begin{abstract}
Aujourd'hui, nous assistons à ce que j'ai proposé d'appeler une vraie " privatisation de la Grande Guerre "z et les familles veulent savoir où l'arrière-grand-père a combattu, où il est éventuellement mort ${ }^{\mathrm{ii}}$. (MCD $2014: 37)$
\end{abstract}

Pour accompagner la doctrine française de 1916 - « l'artillerie dévaste et l'infanterie submerge »-, des batteries seront mises à disposition directe de l'infanterie afin de suivre leur progression au plus près. (MS $2016: 54$ )

En raison de leur représentativité dans le corpus, nous avons donc décidé de nous concentrer sur ces deux phénomènes dont nous avons observé les caractéristiques suivantes : la présence éventuelle de l'origine énonciative, le type et degré de marquage et, enfin, le discours d'escorte. Les analyses seront présentées à partir des fonctions que dénominations et discours directs remplissent au sein des guides touristiques, à partir des cas les plus fréquents.

\title{
4 FORMES ET FONCTIONS DE L'HETEROGENEITE ENONCIATIVE DANS LES GUIDES DE MEMOIRE
}

Les dénominations et les citations en discours direct sont toutes les deux caractérisées par des marques qui pointent ces bribes de texte comme des éléments particuliers: les guillemets, qui en signalent toujours une spécificité énonciative. Enonciateur autre par rapport à l'énonciateur principal du guide, emploi particulier (par exemple figuré), voire prise de distance sont quelques-unes des possibilités que nous avons répertoriées et que nous avons décidé de classer en trois macro-catégories fonctionnelles : garantir l'authenticité du récit historique, susciter le pathos et inscrire les guides dans le patrimoine. Nous allons les examiner dans les paragraphes suivants.

\subsection{Garantir l'authenticité du récit historique}

Les guides de mémoire fondent leur autorité sur la capacité à montrer la maitrise du sujet historique, qui se déploie dans la connaissance des grands événements militaires (batailles, stratégies, commandement, etc.), des lieux et de leurs spécificités (conformation du territoire, dénominations locales, etc.) ainsi que dans le recours à des sources (témoignages, textes officiels, textes littéraires, etc.).

Ce rapport complexe à l'histoire et à l'historiographie peut s'analyser selon trois grands axes, le long desquels il est possible d'établir un continuum :

- Citation plus ou moins explicite de la source ;

- Autorité et fiabilité plus ou moins grande de la source ;

- Adhésion plus ou moins grande du guide par rapport aux propos cités. 
Les deux premiers axes sont bien évidemment reliés par une corrélation étroite : plus la source émane d'une autorité, plus celle-ci sera citée ; toutefois, la question de l'adhésion est bien plus complexe puisqu'il s'agit de vérifier le type d'énonciateur et le moment de l'énonciation, Michelin allant jusqu'à prendre les distances des versions antérieures de son propre guide.

Il existe tout un ensemble de dénominations et d'expressions entourées de guillemets qui font partie d'un fond commun de connaissances, d'une doxa largement partagée et d'un patrimoine du récit national, dont nous allons traiter de manière plus spécifique dans le paragraphe 4.3 .

Ce cimetière-mémorial honore les 13482 soldats britanniques tombés dans le secteur entre octobre 1914 et septembre 1915, sur ce qu'on appelle aujourd'hui «le front oublié » les forces alliées étant alors concentrées dans les régions d'Ypres et de l'Artois. (RCM $2015: 35$ )

L'Entre-deux-guerres nourrit alors une abondante littérature patriotique et nationaliste sur les provinces «perdues » et la « ligne bleue des Vosges ». (MA $2014: 42$ )

Ni les nombreux « on » sujets de l'énonciation, ni les « abondante littérature patriotique » et autres sources sont identifiées et il est impossible pour le lecteur de vérifier l'authenticité de ces informations. Cette stratégie non seulement s'appuie, ainsi que nous venons de l'écrire, sur un fond commun de connaissances partagées, mais elle semble contribuer aussi à imposer d'emblée l'autorité du guide qui fait passer ces expressions pour des évidences, à côté de citations pourvues de références bibliographiques ou du moins de sources.

Il s'agit pour la plupart de dénominations concernant l'activité militaire qui permettent aussi, parfois, une prise de distance a posteriori par rapport à une source non citée, à une « idée fausse » que le guide tente de corriger :

Les Français attaquent en Lorraine pour reconquérir le plus rapidement possible les « provinces perdues ». [...] C'est ce qui est souvent décrit sous l'appellation fallacieuse de « course à la mer ». (MCD $2014: 29)$

Parfois, les éléments guillemétés ne sont pas clairement attribuables à une source, comme dans le cas suivant où l'expression " attaques de grignotage » aurait pu être prononcée par Joffre ou bien cette expression aurait pu être attribuée par la doxa à ce type d'attaque. Nous n'avons donc pas comptabilisé ces exemples :

Après la première bataille de la Marne, en septembre 1914, se sont succédé les attaques de "grignotage » lancées par Joffre à l'hiver 1914-1915, l'immense offensive ratée du 25 septembre 1915, la reprise à l'ennemi du massif de Moronvilliers en avril et mai 1917, la résistance face à l'offensive allemande de la Friedensturm par la $4^{\mathrm{e}}$ armée du général Gouraud, initiatrice de la deuxième bataille de la Marne, et enfin l'offensive victorieuse franco-américaine du 26 septembre 1918 qui libère Sommepy et se termine le 11 novembre à Sedan (MMC 2014 : 144)

Quant aux citations, si l'on regarde tout d'abord celles de membres de l'état-major français ou d'hommes politiques, nous relevons les chiffres suivants:

\begin{tabular}{|l|l|l|l|l|l|l|}
\hline & \multicolumn{2}{|c|}{ Français } & \multicolumn{2}{c|}{ Allemands } & \multicolumn{2}{c|}{ Britanniques et américains } \\
\hline & Noms cités & citations & Noms cités & citations & Noms cités & citations \\
\hline Michelin & 17 & 28 & 2 & 4 & 3 & 3 \\
\hline Routard & 4 & 8 & 5 & 7 & 6 & 27 \\
\hline Petit Futé & 3 & 4 & 3 & 3 & 1 & 1 \\
\hline
\end{tabular}


Au-delà de la différence en termes de quantité de texte, ce qui ressort clairement de ce schéma est la place prépondérante accordée par Michelin au commandement français et par Routard aux Britanniques et Américains, ce qui peut être expliqué par la présence parmi les guides Routard d'un volume consacré au débarquement en Normandie. Quoi qu'il en soit, il ressort quand-même de ce portrait une attitude franco-centrée de Michelin par rapport aux deux autres maisons d'édition. Notons par ailleurs que le Petit Futé est le seul à citer une voix féminine, celle de Marie-Madeleine Fourcade, une résistante (PF 2016 : 82).

Il est aussi possible de retrouver la même citation dans deux guides différents :

C'est à ce moment crucial que Pétain lance son célèbre : " On les aura! » (RCM $2015: 140$ )

Cette offensive est encore un échec et le général Pétain peut galvaniser ses troupes avec cette phrase devenue célèbre :« Courage ... On les aura ! » (MVAS $2016: 72$ )

Les citations qui s'appuient sur des sources fiables et faisant autorité sont nombreuses et sont ainsi distribuées : 10 pour Michelin, 8 pour Routard, 1 pour Petit Futé. Contrairement à ce à quoi on aurait pu s'attendre, les références officielles sont proportionnellement plus nombreuses dans le Routard (3 guides) que chez Michelin (6) (malheureusement les chiffres du Petit Futé sont difficilement significatifs sur un seul guide). Toutefois, c'est Michelin qui présente ses sources en fournissant des détails bibliographiques :

Le premier rapport de la commission parlementaire instituée en vue de constater les actes commis par l'ennemi en violation du droit des gens fut publié au Journal officiel le 8 janvier 1915. Le rapport précise, en ce qui concerne la région, que « dans la plupart des communes de la Marne, des otages ont été emmenés et beaucoup d'entre eux n'ont pas reparu ».

(MMC $2014: 109$ )

Les guides de 1919 s'appuyaient en effet largement sur les déclarations de l'état-major, mais aussi sur les Journaux de Marche et des Opérations, qui constituent la principale source d'information sur les opérations militaires (Kottelat $2015: 266$ ).

Parfois, les textes cités sont même reproduits et donnent lieu à des citations enchâssées comme celle de MAM 2014 : 235, où le guide contemporain cite le guide 1919 qui cite à son tour une affiche de 1916 à propos de l' "affaire Bloch », un jeune espion français fusillé par les Allemands.

Cette page montre clairement les «strates discursives» (Kottelat 2015: 168) et le traitement que les guides contemporains font de leurs ancêtres, ici le guide de Colmariii auquel le guide de 2014 adhère dans ce cas complètement. Or, il n'est est pas toujours ainsi. Chez Michelin, en effet, la prise de distance se fait surtout par rapport aux anciennes version des guides de mémoire, comme dans les exemples suivants :

\section{A NOTER}

Dans cette narration de combats sanglants autour d'Esternay, la différence est flagrante entre le respect du narrateur pour les sépultures des officiers allemands, photographiées, et les détails sordides des exactions commises par les troupes d'occupation. (MMC $2014: 97$ )

\section{A NOTER}

Le guide de l'époque insiste sur le quadrillage du terrain par les artilleurs allemands qui ont pu ajuster les positions françaises. Décimées, nos troupes subissent alors une contre-attaque mue par un enthousiasme confinant à « l'ivresse ». Puisqu'à l'impossible nul n'est tenu, il faudra 
choisir la retraite protégée par « une héroïque arrière-garde ». Quant à l'évacuation de Sarrebourg, elle se fera baïonnette au canon et au son de la Marche Lorraine. (MAM 2014 : 89)

Les guides prennent bien sûr les distances par rapport au discours nazi et pétainiste dans le RD 2014 et le PF 2016 :

On y verra des tickets de rationnement, un certificat de «non appartenance à la race juive » (!) et diverses coupures de journaux ..., sous l'Occupation. Edifiant, par exemple, les déclarations de Pétain pour qui les «lâches attentats » (entendez ceux des résistants) « font horreur à toute la France ». Côté militaire, une importante collection de mines, obus, bombes ... Reconstitution d'une "scène de plage» du 6 juin : mannequins en uniforme au milieu d'obstacles. (RD $2014: 48$ )

On apprend aussi que cette fameuse lettre $\mathrm{V}$ - habituellement au sens de Versuch (prototype) - signifie ici Vergeltung que l'on peut traduire par « représailles », un mot récurrent dans la rhétorique SS. (PF 2016 : 167)

Quant aux guides de la Grande Guerre, la prise de distance se fait surtout face au discours nationaliste et anti-allemand chez Michelin :

«L'ALLEMAGNE PAIERA ! » Le discours de Raymond Poincaré lors de l'inauguration du monument de la Haute-Chevauchée, en juillet 1922, est marqué par une forte tonalité nationaliste. (MVAS $2016: 130$ )

ainsi qu'aux condamnations sommaires des «traitres de la patrie » (vrais ou présumés déserteurs, embusqués et autres) pour Routard:

Une simple pierre dressée au bord du chemin à la mémoire de Lucien Bersot, fusillé pour avoir refusé de porter le pantalon maculé de sang d'un de ses camarades mort au combat, devenue "l'affaire du pantalon rouge ", et de Léonard Leymarie, fusillé pour un « abandon de poste en présence de l'ennemi » jamais prouvé. (RP $2013: 26$ )

Soulignons aussi que cette mémoire imprégnée de «politiquement correct 》 (Kottelat 2015 : 270, 275-276) se doit d'être inclusive. Voilà pourquoi les guides rappellent, entre autres, les troupes coloniales, les travailleurs chinois et les soldats amérindiens employés pour l'encodage des messages.

\subsection{Pathos}

Si on peut affirmer que tous les exemples analysés construisent en quelque sorte un éthos d'expert des éditeurs et rendent le discours historique plus fiable, dans de très nombreux cas, ces fonctions sont côtoyées par l'inscription en discours d'une dimension pathémique, ce qui n'est d'ailleurs pas étonnant étant donné que les liens entre ces deux dimensions de l'argumentation ont déjà bien été montrés par d'autres études (Amossy, 2014, 2016) : si en effet l'éthos discursif se définit comme "l'image que l'orateur construit de sa propre personne pour assurer sa crédibilité » (Amossy 2014 : 13), pour ce faire, le locuteur peut s'appuyer sur l'inscription en discours d'une dimension pathémique : "l'image projetée par l'orateur ne doit pas seulement susciter chez l'auditoire un jugement de valeur fondé en raison : elle doit aussi parler au cœur, elle doit émouvoir» (Amossy 2016 : 117). Cela est d'autant plus vrai que « la fonction émotive, patente dans les interjections, colore à quelques degrés tous nos propos, aux niveaux phonique, grammatical et lexical » (Jakobson 
1963 : 215). Or, il reste à comprendre comment cette dimension pathémique se greffe sur celle éthotique dans ce corpus particulier et quelles fonctions elle remplit. Il nous semble en effet que dans ces guides on peut identifier trois aspects distincts bien qu'entrelacés. De nombreuses citations et dénominations contribuent à dramatiser certains évènements historiques, en les rendant plus tangibles, à insister sur la dimension tragique des deux conflits, tant par le biais des contenus relatés qu'à travers le discours d'escorte et, enfin, à inscrire une mémoire "prothétique » (Raus 2017 : xii), favorisant l'identification des lecteurs avec les soldats dont les dires sont rapportés.

\subsubsection{Dramatiser les évènements}

Précisons d'abord ce que nous entendons par «dramatisation». Nous employons cette expression au sens large pour cerner des phénomènes discursifs variés qui favorisent une certaine théâtralisation du récit historique et par là, visent à rendre le récit plus tangible, à le rapprocher du monde des lecteurs. Dans ce contexte, notamment, certaines formes de citations et de dénominations concourent, nous semble-t-il, à créer cet effet, comme le montre le passage suivant :

A partir du mois de juin 1917, on voit apparaitre dans le ciel du Chemin des Dames un avion allemand qui à l'aube et au crépuscule survole les tranchées en les mitraillant. Surnommé Fantômas, l'avion est évoqué dans beaucoup de souvenirs de soldats. « Ce matin, comme je m'apprêtais à vouloir dormir, écrit un soldat du $93^{\mathrm{e}} \mathrm{RI}$, on nous a signalé le fameux Fantômas qui faisait sa tournée. Mais il n'a pas été bien méchant ce matin. Il est d'abord passé à moins de $80 \mathrm{~m}$ au-dessus de nous. Il a lâché quelques balles de mitrailleuse, puis nous a bombardés pendant 10 minutes. » Dans une autre lettre, un témoin $344^{\mathrm{e}} \mathrm{RI}$ raconte : « Le fameux aviateur boche Fantômas est ici, nous l'avons vu mais nos meilleurs chasseurs se sauvent dès qu'ils le voient. Jamais je n'ai vu un type aussi culotté que celui-là, ce serait dommage de le buter, c'est fantastique. » (MCD 2014 : 40)

Sur cette scénographie énonciative, caractérisée par un régime énonciatif plutôt objectif, où dominent le pronom on et le présent historique, qui alterne avec l'imparfait et le passé composé du récit des évènements, se greffe la première personne des témoignages des soldats. Le passage d'un régime à l'autre se fait via l'inscription de la dénomination «Fantômas » par le verbe métalinguistique au passé composé surnommer. C'est le point de vue (PDV) des soldats cités que les éditeurs adoptent dans cet énoncé, qui fonctionne à l'instar de discours d'escorte introducteur du DD sans qu'aucun nom d'agent (qui le surnomme «Fantômas »?) ou aucun verbe métalinguistique ne le signale. Le lecteur est ainsi amené à adopter le même PDV des soldats, à communier avec eux, à revivre ces moments dans les tranchées, bref, à partager la situation de communication dans laquelle ces mots ont été écrits. Il arrive parfois, que la description entière de certaines batailles soit confiée à de longues citations littéraires qui, tout en étant des témoignages directs des tranchées, se caractérisent par une certaine verve discursive et un style soigné :

\section{LA FONTENELLE, 22 JUIN 1915}

« De formidables explosions ébranlent l'air : les Allemands ont fait jouer trois mines (... ) Notre tranchée couverte est détruite sur une cinquantaine de mètres. Aussitôt après, un bombardement intense commence : une trentaine de batteries de gros calibre crachent leurs projectiles sur les positions de la cote 627 et le village de la Fontenelle. ( ... ) Les résultats de ces tirs, des plus précis, sont terribles. Les boyaux sont entièrement comblés, les tranchées écrasées et les abris pulvérisés. [...]» Capitaine Ernest Dupuy, La Guerre dans les Vosges, Paris, Payot, 1936. (MAM $2014: 158)$ 
Ce sont alors les écrivains combattants qui sont sollicités dans ces guides pour témoigner le caractère extraordinaire de certains évènements: modalisateurs adjectivaux ( $«$ de formidables explosions », « un bombardement intense» «résultats [...] terribles »), présence de climax rhétoriques («Les boyaux sont entièrement comblés, les tranchées écrasées et les abris pulvérisés »), préférence accordée à la parataxe et ponctuation conférant au discours un rythme rapide concourent à la dramatisation de l'importance de l'enjeu. Cela arrive également dans l'extrait suivant, où le récit du moment de fraternisation avec l'ennemi allemand est confié au témoignage d'un soldat :

Lorsqu'arrive Noël 1914, à plusieurs endroits du front, certains soldats, épuisés par cette hécatombe, n'hésitent pas à sortir des tranchées pour se rencontrer dans le no man's land. « Maintenant, depuis que, pour quelques heures, les hommes d'en face ne sont plus nos ennemis, un peu de joie flotte dans l'air et la neige semble moins froide ", décrit le soldat Renaud. (RCM 2015 : 14-15)

Déictiques temporel et personnels et perceptions physiques du soldat rendent le récit plus vif : comme si le lecteur était là et pouvait participer à la situation de communication, en même temps que le combattant.

\subsubsection{Insister sur la dimension tragique}

D'autres cas d'hétérogénéité énonciative montrent davantage la volonté des éditeurs d'insister sur le caractère tragique des conflits. A cet effet peut concourir l'inscription en discours de chiffres montrant le nombre extrêmement élevé de morts et de blessés, comme cela arrive dans les exemples suivants, tant dans le contenu des citations que dans le discours accompagnant les dénominations :

À $500 \mathrm{~m}$ d'altitude, ce pôle défensif sur la route d'Épinal, surnommé le « col de la mort» ou le «trou de l'Enfer», a changé plusieurs fois de main et englouti 4000 Français durant les premières semaines de la Grande Guerre. (MAM 2014 : 126-128)

En 2008, le Dictionnaire de la Grande Guerre (sous la direction de F. Cochet et R. Porte) avance que « Verdun voit disparaitre près de 600

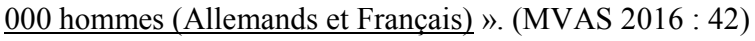

215 personnes mortes suite à leur internement au camp de concentration du Vernet d'Ariège [...]. Le dernier convoi - surnommé le «train fantôme »est parti en juin 1944 : les 403 derniers internés ont été évacués vers Toulouse. (PF $2016: 154)$

L'emploi des chiffres, argument relevant typiquement d'une argumentation s'appuyant sur le logos (Perelman et al., 2008), remplit dans ces extraits deux fonctions complémentaires : d'un côté, il répond à la nécessité de tenir un discours historique précis et fiable, de l'autre, il permet d'insister sur les proportions extraordinaires des pertes pendant les conflits.

Un autre procédé fort fréquent concerne l'emploi de dénominations dysphoriques des lieux ou des évènements relatés. Ces dénominations sont parfois attribuées à des sources énonciatives dignes de confiance, comme dans les cas suivants :

Les Allemands ont détruit méthodiquement les usines, dynamité et inondé les puits de mine. À la fin de l'année 1918, les journalistes parisiens 
découvrent les paysages ravagés entre la Somme et Lille et parlent de «L'Enfer du Nord» (MFA $2013: 46$ )

Signalé par une croix de $20 \mathrm{~m}$ de haut sur $5,25 \mathrm{~m}$ de large, les poilus surnommaient cette colline « la mangeuse d'hommes » ou «la montagne de la Mort $»$. (PF $2016: 60$ )

Néanmoins, dans la plupart des extraits, ces dénominations ne sont pas reliées à des sources énonciatives. Ce sont donc des on-dire qui sont relatés, soit de l'époque contemporaine aux guerres soit postérieurs :

À côté de l'ancien moulin de Sarraltroff, en bordure de la route menant à Dolving par la ferme du Sarrewald, une autre stèle rappelle que dans $\underline{\text { la }}$ «tranchée de la mort », une compagnie du $27^{\mathrm{e}} \mathrm{RI}$ fut presque totalement anéantie au cours de l'offensive française du 19 août. (MAM $2014: 85$ )

Le sort des «gueules cassées » a pleinement été révélé par le roman $L a$ Chambre des Officiers paru en 1999 de Marc Dugain puis porté à l'écran en 2001 par François Dupeyron. Les gueules cassées de la guerre 1418 durent tant bien que mal revenir à la vie civile mais pour certains ce retour s'avéra impossible et ces mutilés de la face mais aussi du cerveau se retrouvèrent dans des hôpitaux psychiatriques ou des institutions spécialisées. (PF 2016 : 64)

L'opacité de l'origine énonciative ne fait qu'augmenter le caractère présumé partagé des contenus ainsi véhiculés qui, par-là, sont inscrits dans la mémoire collective des locuteurs. Le récit des conditions de vie des soldats constitue un autre moyen par lequel les éditeurs visent l'inscription en discours d'une dimension pathémique : les écrits des poilus sont alors mobilisés pour montrer le caractère dramatique de leur expérience au front.

Le gaz propagé depuis les lignes allemandes dans le secteur de Steenstraat et Langemark fait des ravages chez les soldats pris par surprise. " Des hommes se roulaient par terre, convulsés, toussant, vomissant, crachant le sang », raconte un soldat français. (RCM $2014: 15$ )

Dans cet extrait d'une lettre écrite par un "soldat français ", la charge émotive est portée essentiellement par des lexèmes dénotant les réactions des soldats dans une situation limite. Les guides n'hésitent en effet pas à citer des extraits prétendant mettre en scène les dures conditions de vie des soldats au front ainsi que leurs émotions, qui ne font qu'exacerber le caractère dysphorique de la narration :

\section{TÉMOIGNAGE DU CAPITAINE BELMONT}

« C'est près du sommet de ce fameux Linge où nous sommes que le $11^{\mathrm{e}}$ est, depuis le 5 août, au contact étroit avec des Boches [...]. Entre ces deux lignes, le terrain est devenu un charnier. Des corps de chasseurs tombés au cours d'attaques successives, des corps d'Allemands tués par nos fusils dans les dernières contre-attaques gisent étendus dans toutes les positions, au milieu des fils de fer tordus et des sapins abattus. À certains moments, l'air soulève des odeurs atroces. » (MAM 2014 : 216)

\subsection{Patrimonialisation}

De nombreuses instances de discours rapporté sont intégrées par les guides dans le patrimoine commun qui concourt à composer le récit national: citations d'écrivains, inscriptions sur les monuments et les tombeaux, etc. De cette manière, les ouvrages 
littéraires contribuent autant que les monuments et les restes matériels à cette patrimonialisation du passé traumatique. Michelin est le champion des citations littéraires : on y trouve 17 citations tirés de la littérature française et 13 des autres littératures, notamment anglaise. Dans le MS 2016, un encadré de deux pages (66-67) est entièrement consacré aux « écrivains de la bataille de la Somme » :

Si l'expérience combattante est relatée différemment par des écrivains aussi opposés que Ernst Jünger ou Henri Barbusse, ces derniers pourront se rejoindre dans une appréciation similaire de la société, en déclarant, chacun à leur tour : «nous sommes des machines à oublier ». (MS $2016: 66$ )

La littérature appartient au patrimoine national, mais aussi à celui de l'humanité entière permettant ainsi de mettre en œuvre une rhétorique de la fraternité et de la réconciliation. Le Routard présente quant à lui quatre citations d'auteurs français ou francophone (Senghor), une seule d'un auteur britannique, alors que le Petit Futé se limite à trois citations françaises.

De leur côté, les inscriptions sont très représentées parmi les expressions guillemetées, dont l'importance symbolique est parfois ouvertement soulignée :

Situé à l'angle d'un carrefour, masqué par une haie qui le rend invisible, le monument érigé à la mémoire des « deux premiers gars du $26^{\mathrm{e}}$ » tombés en pénétrant en terre lorraine acquiert valeur de symbole. (MAM 2014 : 78)

Cette importance symbolique s'accompagne d'une valeur plus concrète de témoignage historique faisant état de tributs mémoriels réalisés par des anciens combattants :

Une belle œuvre du sculpteur Fischer y trône, dédiée " à ceux qui n'ont pas de tombe ". A côté du monument du Point X, au bord d'un entonnoir, se trouve un petit mur de pierre dans lequel est fixée une plaque qui porte, en plus d'une croix de guerre, l'inscription suivante : " 302 e RI, 20 septembre 1914, 21 mars 1915. Les Anciens des 302 ${ }^{\mathrm{e}}$ et 102- RI". (RCM $2015: 175$ )

Mais les fonctions de ces inscriptions sont extrêmement diversifiées : elles peuvent marquer une prise de distance par rapport au discours patriotique :

A la fin de la guerre, de nombreuses familles se déchirent autour du sort réservé à leurs défunts : les laisser reposer dans des cimetières allemands ou les enterrer sous des croix où figure "Mort pour la France»? En Alsace et en Lorraine autrefois occupée, l'inscription « Morts pour la France» est dans la plupart des cas remplacée par des formules plus neutres telles que «La commune à ses enfants", « nos victimes des deux guerres» ou encore plus simplement «A nos morts», les monuments regroupant parfois sous une même stèle le nom de soldats originaires du village, quel que soit leur uniforme. (RCM 2015 : 186)

opérer un rapprochement envers l'ennemi d'antan dans la douleur commune provoquée par la mort et le deuil (même si cet exemple peut avoir une nature fort ambiguë, du moins pour nous qui lisons tout cela à partir d'une perspective post-nazi) :

Le mur en brique comporte une arche portant l'inscription «Zur Ehre der für Kaiser und Reich gefallenen Sëhne Deutschlands » (« En l'honneur des fils du Reich tombés pour le Kaiser et l'Empire »). (MS 2016 : 71)

ou encore, justement, souligner la continuité entre les deux guerres : 
L'inscription des dates de la Seconde Guerre mondiale sur la pierre nue rappelle également que l'on se trouve dans une zone de combats meurtrière pour les Américains en 1944. (MAM $2014: 66$ )

Cette opération de patrimonialisation des bribes de texte, que ce soient des pages de littérature ou des épitaphes, que l'on y adhère ou que l'on en prenne les distances, contribue à renforcer, voire à créer, ce fond culturel commun du récit national, qui va à son tour générer un engouement pour les lieux de mémoire et donc pour le tourisme de mémoire.

\section{CONCLUSION}

Au terme de ce survol, il nous semble pouvoir affirmer que l'hétérogénéité énonciative joue un rôle central dans la construction du discours touristique de la mémoire des conflits mondiaux du $\mathrm{XX}^{\mathrm{e}}$ siècle. La présence massive du discours autre, tant sous la forme de citations que sous celle de dénominations, caractérise tous les guides analysés, en dépit des différences traditionnelles entre guides pratiques, les Routard et le Petit Futé et guides culturels, les Michelin. Si la première typologie semble privilégier la voix des soldats simples, souvent anonymes, alors que dans guides Michelin les citations proviennent plus volontiers de textes officiels ou, au moins, de soldats gradés dont on explicite l'identité, l'hétérogénéité énonciative dans ce dernier type de guides apparait comme fondant même la macrostructure des textes et en justifie la publication. Un va-et-vient continu entre la toute première édition de ces guides et celle d'aujourd'hui ponctue les textes et permet tantôt de s'y appuyer tantôt d'en prendre les distances, montrant ainsi jusqu'à quel point le discours de la mémoire se modifie au fil du temps, selon les aléas de l'histoire et les contextes socioculturels dans lesquels il circule. Preuve en est le difficile équilibre dont témoignent les guides analysés entre la nécessité de maintenir une distance par rapport au discours patriotique tout comme par rapport au discours antimilitariste. C'est pourquoi il n'y a que rarement une prise de position nette, exception faite pour les discours "extrêmes" (pétainiste et nazi fondamentalement), bien que le lecteur soit toujours laissé à sa propre interprétation. Une comparaison de ce type de discours avec ceux de la presse, des réseaux sociaux et des représentations artistiques et littéraires foisonnant ces dernières années permettrait dès lors de circonscrire tant les phénomènes discursifs communs aux différents types de discours tant les caractéristiques permettant de les distinguer les uns des autres afin de saisir les procédés régissant la construction de la mémoire collective à l'époque contemporaine.

\section{Références bibliographiques}

Amossy R. 2016. "Dimension rationnelle et dimension affective de l'ethos », in Rinn M. (dir.), Émotions et Discours. L'usage des passions dans la langue, Rennes: Presses universitaires de Rennes, 113-125.

Amossy R. 2014. «L'éthos et ses doubles contemporains Perspectives disciplinaires ». Langage \& société, 149, 13-30.

Authier-Revuz J. 1995. Ces mots qui ne vont pas de soi. Boucles réflexives et non-cö̈ncidences du dire, $2 \mathrm{t}$, Paris : Larousse.

Authier-Revuz J. 1984. «Hétérogénéité(s) énonciative(s)». Langages, 73, 98-111. En ligne : https://www.persee.fr/doc/lgge_0458-726x_1984_num_19_73_1167

Barthelmebs-Raguin H., Komur-Thilloy G., Lopez-Muñoz J. M., Marnette S., Rosier L. (dir.). 2018. Le Discours rapporté. Temporalité, histoire, mémoire et patrimoine discursif. Paris : Classiques Garnier. 
Hertzog, A. 2012. «Tourisme de mémoire et imaginaire touristique des champs de bataille ». Via[at], 1. En ligne : https://journals.openedition.org/viatourism/1276 [consulté le 03/01/2020].

Kerbrat-Orecchioni, C. 2004. "Suivez le guide ! Les modalités de l'invitation au voyage dans les guides touristiques : l'exemple de l'"île d'Aphrodite" ", in Baider, F., Burger, M., Goutsos, D. (dir.), La communication touristique. Approches discursives de l'identité et de l'altérité, Paris : L'Harmattan, 133-150.

Kottelat, P. 2015. «Du tourisme de pèlerinage au tourisme de mémoire : la réédition des Guides des champs de bataille Michelin », in Paissa P., Rigat F., Vittoz M.-B. (dir.), Dans l'amour des mots. Chorale(s) pour Mariagrazia, Alessandria, Edizioni dell'Orso, 265-277.

Longhi, J. 2014. «L'usage de la dénomination, la dénomination par l'usage ». Daval R., Hilgert E., Nicklas T., Thomières D. Sens, formes, langage. Contributions en l'honneur de Pierre Frath, EPURE, 269- 282.

Lugrin, G. 2004. « La construction des icônes identitaires des lieux touristiques: Chypre dans les publicités touristiques de langue française », in Baider, F., Burger, M., Goutsos, D. (dir.), La communication touristique. Approches discursives de L'identité et de l'altérité. Paris : L'Harmattan, 235-256.

Jakobson, R. 1963. Essais de linguistique générale. Paris: Minuit.

Perelman, C., Olbrechts-Tyteca L. 2008. Traité de l'argumentation : La nouvelle rhétorique. Bruxelles : Editions de l'Université de Bruxelles.

Raus, R. 2017. « Préface », in Raus, R., Cappelli G., Flinz C. (dir.), Le Guide touristique : Lieu de rencontre entre lexique et images du patrimoine culturel. Firenze : Firenze University Press, viixiv.

Rebeyrolles, J. 2004. "L'acte définitoire dans les guides touristiques », in Baider, F., Burger, M., Goutsos, D. (dir.), La communication touristique. Approches discursives de l'identité et de l'altérité, Paris : L'Harmattan, 173-187.

Rosier, L. 2008. Le discours rapporté en français, Paris : Ophrys.

\section{Guides Michelin}

Les Champs de bataille. La Marne et la Champagne (2014).

Les Champs de bataille. Verdun, Argonne, Saint-Mihiel (2016).

Les Champs de bataille. Alsace Moselle : Les combats des Vosges (2014).

Les Champs de bataille. Flandres Artois : Ypres, Nord, Pas-de-Calais (2013).

Les Champs de bataille. Le Chemin des Dames : Aine, Oise (2014).

Les Champs de bataille. Somme: Amiens, Péronne, Albert (2016).

\section{Le Routard}

Picardie 14-18. Centenaire d'un conflit mondial (2013).

Le Débarquement \& la bataille de Normandie (2014).

Grande Guerre 14-18. Les chemins de mémoire. Des Flandres aux Vosges (2015).

\section{Petit Futé}

Guide des lieux de mémoire (2016).

\footnotetext{
${ }^{i}$ Pour plus de renseignements sur ce projet porté par le Département de Langues et cultures étrangères de Gênes, on peut consulter le blog à l'adresse suivante : https://memwarunige.hypotheses.org/

ii Nous soulignons les parties commentées dans les extraits analysés.

iii Pour une description des guides de mémoires Michelin publiés dans l'immédiat après-guerre voir Kottelat 2015.
} 